\title{
Numerical Analysis of the Resolution of Surface Electrocardiographic Lead Systems
}

\author{
Jesús Requena-Carrión ${ }^{1}$, Juho Väisänen ${ }^{2}$, José Luis Rojo-Álvarez ${ }^{1}$, \\ Jari Hyttinen $^{2}$, Felipe Alonso-Atienza ${ }^{1}$, and Jaakko Malmivuo ${ }^{2}$ \\ ${ }^{1}$ Departamento de Teoría de la Señal y Comunicaciones, \\ Universidad Rey Juan Carlos, Fuenlabrada, Spain \\ jesus@tsc.uc3m.es \\ ${ }^{2}$ Ragnar Granit Institute, \\ Tampere University of Technology, Tampere, Finland
}

\begin{abstract}
Non-invasive electrocardiographic (ECG) techniques for assessing the electrical activity of selected regions within the cardiac muscle can benefit from suitable positioning of surface electrodes. This positioning is usually guided heuristically and complemented by clinical and experimental studies, but there is a lack of general methods to characterize quantitatively the ability of a given electrode configuration to focus on selected regions of the heart. In this study we explore an approach to the characterization of the resolution of surface ECG systems based on the concept of Resolution Mass (RM). By integrating bioelectric signal modeling and numerical methods, we explore, in an application example, the location and size of the RM for a multielectrode ECG system. The concept of RM combined with bioelectric signal modeling and numerical methods constitutes a powerful tool to investigate the resolution properties of surface ECG systems.
\end{abstract}

\section{Introduction}

Surface electrocardiogram (ECG) is one of the most common diagnostic methods for cardiac diseases. In many diseases, such as ischemia and infarction, the diagnosis involves an estimation of the localization and extension of an abnormal region within the heart. This region is usually determined by detecting well known anomalies in the morphology of conventional 12-lead ECG signals. Diagnostic methods based on ECG techniques can be further refined by an adequate choice of the position and configuration of surface electrodes [1] 2]. From the general principles of Bioelectromagnetism, it is expected that each electrode configuration focuses on different regions of the heart. Therefore, electrode configurations show distinctive intrinsic resolution properties that can provide us with valuable insight into regional information. Usually, the positioning of surface electrodes is guided heuristically and complemented by means of studies in which extended multielectrode systems are used [3] [4] [5]. Nevertheless, a widely accepted quantitative approach to the study of the resolution properties of electrode configurations is still lacking. As a consequence, there is limited agreement on the nature of these resolution properties. 
The aim of this study is to explore the notion of resolution of surface ECG systems using the concept of Resolution Mass (RM). The RM is defined as the myocardial mass that contributes the most in power terms to the ECG signal. Considering that the heart is the object of our investigation, and that we can only access its state indirectly through ECG signals, we form our approach from two starting points. On the one hand, in order to place the notion of resolution in a quantitative framework, we define a measurement over ECG signals, since they are the only observations. Conventional mean power is a meaningful candidate due to its clear physical meaning. On the other hand, we link ECG signals to the electrical activity of the heart, so that any statement from the previously defined measurement over ECG signals can be extended to the heart itself. This implies modeling ECG signals based on Bioelectromagnetism Theory, according to which bioelectric phenomena arise as a result of the interplay of source and conducting elements in the body [6]. In the context of lead systems, Lead Field Theory is a convenient mathematical formulation from which we can benefit both conceptually and computationally. From these starting points, we approach the notion of resolution by asking what the contribution in power terms of a certain myocardial mass within the heart is to a given ECG signal. We propose to answer it by determining the similarity in mean power between the signals generated by the myocardial mass under investigation and by the whole myocardium, respectively. The knowledge of the contributions of different myocardial masses will, in turn, allow us to decide which one of them can be established as the RM. Since this procedure is not feasible in experimental settings and classical analytical techniques fail in this scenario, numerical analysis will be crucial to this study, by constituting the basic tool to carry what has come to be widely known as experiments in silico.

In the following sections, we firstly introduce our implementation of the model of ECG signals in terms of the dynamics of the heart and the lead fields associated to the electrode configuration, i.e., in terms of source and conducting elements. Secondly, we provide a definition of RM, and we propose a numerical procedure to estimate it. Thirdly, we investigate, as an example, the resolution properties of an ECG system over the ventricles. Finally, we summarize our results and propose future applications of the concept of RM.

\section{Bioelectric Signal Modeling}

In the electric characterization of the human body, two simultaneous features are described, namely its behavior as a source volume and as a conductor volume [6]. A source volume is a tissue that can generate non-conservative electric currents, whereas a conductor volume allows source elements to induce electric potentials in other regions of the body. When such electric potentials are recorded on the skin, a surface ECG signal is obtained. Consequently, any model of ECG signals in terms of the electrical properties of the human body will consist of a description in terms of both cardiac sources and conducting properties of the body. 


\subsection{Modeling the Cardiac Tissue as a Bioelectric Source}

In our implementation we opted for a state machine formulation for the electric activity of cardiac tissue defined previously [7. This formulation is able to reproduce the electric restitution of both action potential duration (APD) and conduction velocity (CV), as well as curvature effects. Cardiac tissue is modeled as a grid of discrete elements characterized by three discrete states, namely, Rest, Refractory 1 and Refractory 2 , and three transitions among them. The excitation of an element, i.e. the transition from Rest to Refractory 1 , is interpreted as a probabilistic event depending on the amount of excitation in its neighborhood and the excitability of the element, which is accessible through the current value of CV. Transitions from Refractory 1 to Rest through Refractory 2 depend on the current value of APD. Both APD and CV values are updated at every new diastolic interval (DI) based on the curves of electric restitution. Transitions among states, at every time instant $n$, are defined as follows:

$$
\begin{gathered}
\text { Rest } \rightarrow \text { Re fractory } 1: P_{\text {exc }}^{j} \propto C V \cdot Q_{\text {exc }}^{j} \\
\text { Refractory } 1 \rightarrow \text { Refractory } 2: n-n_{\text {desp }}^{j} \geq F \cdot A P D \\
\text { Refractory } 2 \rightarrow \text { Rest }: n-n_{\text {desp }}^{j} \geq A P D,
\end{gathered}
$$

where $j$ identifies an excitable element in the grid, $P_{e x c}^{j}$ is the probability of excitation of $j, Q_{e x c}^{j}$ is the amount of excitation within a neighborhood around $j, n_{\text {desp }}^{j}$ is the instant of depolarization of $j$, and $F$ is a scalar representing the fraction of APD that $j$ spends at Refractory 1 while depolarized. Additionally, a membrane voltage is assigned at every time instant. Its value is the usual rest voltage during Rest state, and a standard version of an action potential temporarily scaled by the APD during Refractory 1 and Refractory 2 . Finally, source elements are computed from the voltage differences and conductivities between neighboring elements.

\subsection{Modeling the Body as a Bioelectric Conductor}

Based on the conducting properties of the body, the solution of the forward problem allows us to obtain the electric potential induced by a source element at every location of the body. From the principle of superposition, given a distribution of source elements within the body, the total electric potential can be expressed as the superposition of the electric potentials generated individually by every source element. Nevertheless, when studying signals recorded by lead systems, we are only interested in the electric potentials induced at a finite number of locations in the body. Furthermore, a formulation that explicitly includes the measurement sensitivity distribution of the lead system could be conceptually more powerful. In this context, Lead Field Theory provides an adequate formulation that is equivalent to the forward solution. It has conceptual 
advantages, since it includes in its definition the measurement sensitivity distribution of the lead system, and, if implemented numerically, it is computationally less demanding. According to Lead Field Theory, the signal recorded by a lead system, $z[n]$, can be expressed as a linear combination of source elements in the source volume $V$ as follows:

$$
z[n]=\sum_{V} \frac{1}{\sigma} \frac{1}{I_{r}} \bar{J}_{L} \cdot \bar{J}_{i}[n] .
$$

In this discretized formulation, $I_{r}$ is the reciprocal current, $\bar{J}_{L}$ is the lead current density and constitutes the measurement sensitivity distribution, $\bar{J}_{i}[n]$ denotes the source elements in $V$, and $\sigma$ is the conductivity. Conceptually, this approach allows us to interpret the dynamics of $z[n]$ in terms of the dynamics of those regions of $V$ where the sensitivity of the lead system is highest. Computationally, when simulating $z[n]$, it overcomes the need of solving the forward problem for every source element $\bar{J}_{i}[n]$ at every time instant $n$. Instead, by virtue of the Reciprocity Theorem, $\bar{J}_{L}$ is obtained by solving the forward problem once and for all for a reciprocal current applied to the surface electrode, and then, at every time instant $n, z[n]$ is readily calculated from (2).

\section{Measuring the Resolution}

Let $V_{o}$ and $V_{c}$ be two disjoint subsets of the source volume $V$ such that $V=$ $V_{o} \cup V_{c}$. From (2), we can express the ECG signal $z[n]$ generated by $V$ as the sum of the signals $z_{o}[n]$ and $z_{c}[n]$ induced, respectively, by $V_{o}$ and $V_{c}$ :

$$
\begin{aligned}
z[n] & =\sum_{V} \frac{1}{\sigma} \frac{1}{I_{r}} \bar{J}_{L} \cdot \bar{J}_{i}[n] \\
& =\sum_{V_{o}} \frac{1}{\sigma} \frac{1}{I_{r}} \bar{J}_{L} \cdot \bar{J}_{i}[n]+\sum_{V_{c}} \frac{1}{\sigma} \frac{1}{I_{r}} \bar{J}_{L} \cdot \bar{J}_{i}[n] \\
& =z_{o}[n]+z_{c}[n] .
\end{aligned}
$$

If we measure the similarity between $z[n]$ and $z_{o}[n]$ based on Mean Square Difference (MSD), we obtain:

$$
\begin{aligned}
\operatorname{MSD}\left\{V_{o}\right\} & =E\left[\left(z[n]-z_{o}[n]\right)^{2}\right] \\
& =E\left[\left(z_{c}[n]\right)^{2}\right] \\
& =P_{z_{c} z_{c}},
\end{aligned}
$$

where $E$ denotes statistical mean. Thus, $M S D\left\{V_{o}\right\}$ is a measurement of the error involved when approximating ECG signal $z[n]$ to $z_{o}[n]$. If we normalize 
$M S D\left\{V_{o}\right\}$ by the mean power of $z[n], P_{z z}=E\left[(z[n])^{2}\right]$, we can obtain a more convenient measurement of similarity relative to the mean power of $z[n]$ :

$$
\begin{aligned}
M S D_{n}\left\{V_{o}\right\} & =\frac{M S D\left\{V_{o}\right\}}{P_{z z}} \\
& =\frac{P_{z_{c} z_{c}}}{P_{z z}} .
\end{aligned}
$$

We define the RM at level $\alpha, R M_{\alpha}$, of a surface ECG as the smallest region $V_{o}$ within the heart that involves a $M S D_{n}$ less than $\alpha$ when approximating at the recording site the electric potential generated by the whole heart to the electric potential generated by the RM, i.e.,

$$
R M_{\alpha}=\arg \min _{V_{o}}\left\{\operatorname{Size}\left(V_{o}\right)\right\}, \forall V_{o} / M S D_{n}\left\{V_{o}\right\} \leq \alpha
$$

In other words, we are measuring the resolution properties of an ECG system in terms of the power of the component $z_{c}$ of the ECG signal generated in the region excluded from the RM, $V_{c}$. The RM has, thus, a clear physical meaning and it is suitable to carry out fair comparisons between electrode systems, since it is obtained from a measurement defined over the ECG signal. In this study, we investigate two properties of the RM, namely, its mass center and its size. The mass center of a RM allows us to determine the location of the RM within the whole source volume. The size is a meaningful parameter to describe the scope of the RM, since it informs us of the ability of the electrode system to focus on selected regions of a given size within the source volume.

We used numerical methods to estimate $R M_{\alpha}$ in two ways. On the one hand, we obtained the lead field based on Finite Difference Methods (FDM) featuring the Visible Human Man model (VHM), by calculating the electric potential generated by a reciprocal current applied to the surface electrodes 8 . According to the Reciprocity Theorem, generated lead current fields $\left(\bar{J}_{L}\right)$ raised by the reciprocal current $\left(I_{r}\right)$ corresponds to the lead field. On the other hand, we generated implicitly the dynamics of $\bar{J}_{i}[n]$ using a computer, i.e., through numerical simulations. Then, by implementing the model of cardiac tissue as defined earlier,

we simulated $\bar{J}_{i}[n]$ and, for different choices of $V_{o}$, from (3) we generated $z_{o}[n]$, $z_{c}[n]$ and $z[n]$. Subsequently, from (4) and (5), we estimated $M S D_{n}\left\{V_{o}\right\}$ and, finally, for every $\alpha$ we determined $R M_{\alpha}$ according to (6).

\section{Example}

The characterization of the resolution properties of a surface ECG system based on the determination of $R M_{\alpha}$ is exemplified here for a 117 unipolar electrode ECG system. From this system, a total of 18 unipolar electrodes distributed around the thorax on the cross section located at the fourth intercostal space were investigated (Figure 1); among them, electrodes numbered 22 and 37 correspond respectively, to precordial leads $V_{1}$ and $V_{2}$ in conventional 12-lead ECG. 


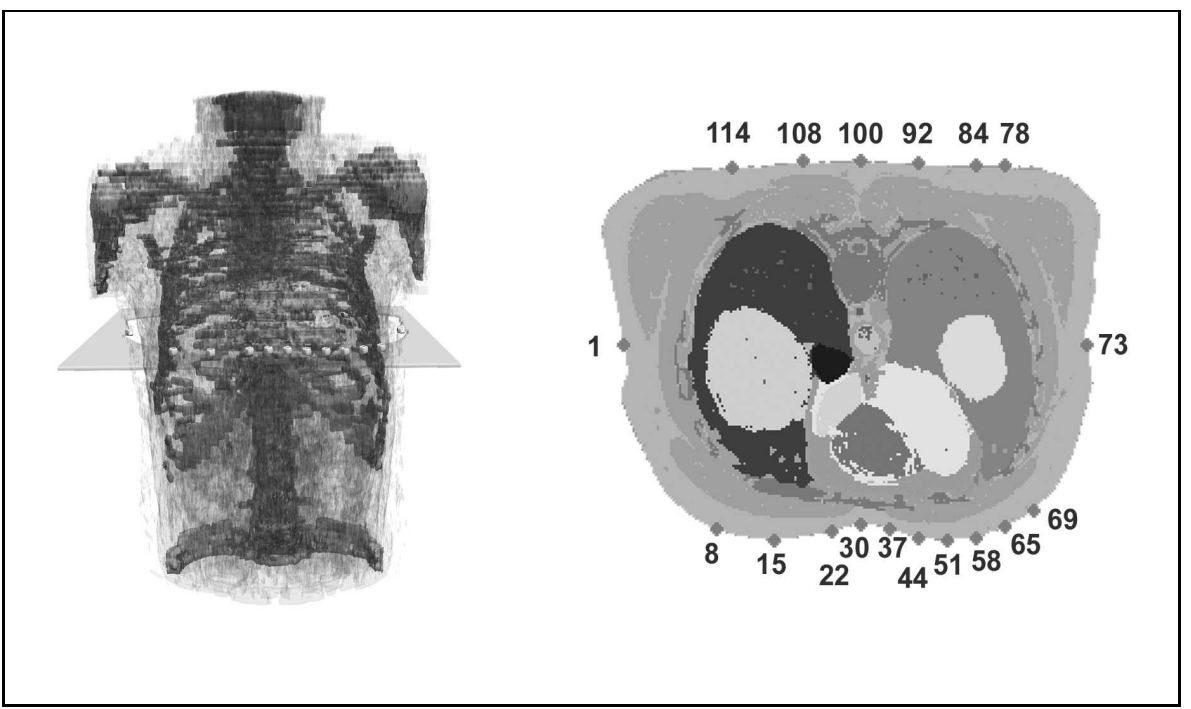

Fig. 1. Thorax model as described by the VHM dataset. Studied surface electrodes are positioned around the thorax on a cross section located at the fourth intercostal space.

We studied $R M_{\alpha}$ within the ventricles (Figure 2) as a function of $\alpha$ and the position of the electrodes. For this purpose, we calculated numerically $\bar{J}_{L}$ as described earlier. Then, we programmed the state machine of electric activity of cardiac tissue, and we simulated the stimulation of the ventricular apex at 60 beats per minute during 8 seconds. Progressively larger source volumes $V_{o}$ were chosen to synthesize signals $z[n], z_{o}[n]$ and $z_{c}[n]$, from which corresponding $M S D_{n}\left\{V_{o}\right\}$ were obtained. Finally, for several values of $\alpha, R M_{\alpha}$ was determined according to (6).

Figure 3 shows estimated $R M_{\alpha}$ for electrode 37 (precordial lead V1 in 12-lead ECG) and three values of $\alpha: 0.1,0.2$ and 0.5 . As it is expected, larger $\alpha$ values lead to a smaller size of $R M_{\alpha}$, since $\alpha$ measures the $M S D_{n}$ involved when approximating the signal induced by the ventricles to the signal induced by $R M_{\alpha}$. Furthermore, we can observe that $R M_{0.1}, R M_{0.2}$ and $R M_{0.5}$ are geometrically close to the position of electrode 37 , which agrees with the fact that the measurement sensitivity decreases with the distance to the electrode. This observation was confirmed for every electrode.

In Table 1 the sizes of $R M_{0.1}, R M_{0.2}$ and $R M_{0.5}$ relative to the size of the ventricles can be explored for every electrode. Apart from the decrease in size for larger values of $\alpha$ that was noted earlier, two qualitatively different sets of results are relevant, depending on whether electrodes are positioned on the anterior or the posterior chest. In general, $R M_{\alpha}$ for anterior electrodes is comparatively smaller than for posterior electrodes. These results can be explained by the fact that distances from the skin surface to the ventricles are greater on the posterior than on the anterior chest (see Figure 1). For example, while the size of $R M_{0.5}$ 


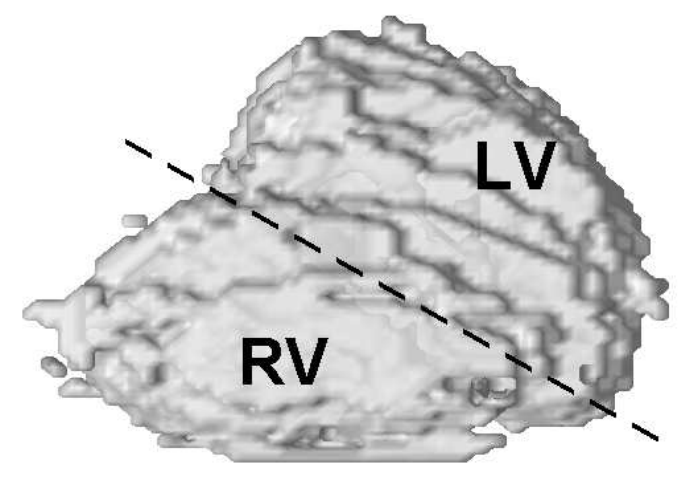

Fig. 2. Superior view of the ventricular myocardial mass as described by the VHM dataset. Right ventricle $(\mathrm{RM})$ and left ventricle $(\mathrm{LV})$ are, respectively, the masses below and above the dashed line.

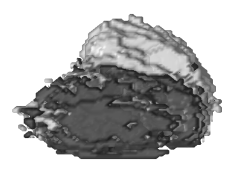

(a) $\alpha=0.1$

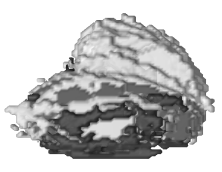

(b) $\alpha=0.2$

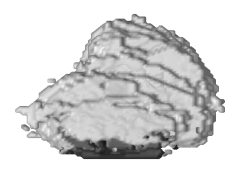

(c) $\alpha=0.5$

Fig. 3. Superior view of the ventricles (light gray) in the VHM model and estimated $R M_{\alpha}$ (dark gray) of electrode 37 (precordial lead V1 in 12-lead ECG) for different values of $\alpha$. We can observe that larger $\alpha$ values lead to smaller $R M_{\alpha}$.

for electrode 84 is roughly $83 \%$ of the size of the ventricles, for electrode 37 it is $13 \%$. Therefore, the ability of unipolar electrodes on the posterior chest to focus on small regions of the ventricles will be, in general, poorer than that of electrodes on the anterior chest. It is equally remarkable that the amount of 
Table 1. Size of $R M_{\alpha}$ (relative to the size of the ventricles), for $\alpha: 0.1,0.2$ and 0.5

\begin{tabular}{||l||c|c|c|c|c|c|c|c|c|c|c|c|c|c|c|c|c|c||}
\hline Electrode & 1 & 8 & 15 & 22 & 30 & 37 & 44 & 51 & 58 & 65 & 69 & 73 & 78 & 84 & 92 & 100 & 108 & 114 \\
\hline \hline$R M_{0.1}(\%)$ & 55 & 84 & 85 & 73 & 63 & 65 & 64 & 63 & 58 & 54 & 52 & 57 & 87 & 90 & 84 & 69 & 54 & 47 \\
\hline$R M_{0.2}(\%)$ & 25 & 75 & 73 & 53 & 32 & 47 & 44 & 43 & 40 & 38 & 37 & 47 & 85 & 89 & 79 & 58 & 43 & 35 \\
\hline$R M_{0.5}(\%)$ & 10 & 10 & 13 & 2 & 1 & 13 & 22 & 22 & 19 & 17 & 16 & 21 & 75 & 83 & 67 & 28 & 16 & 11 \\
\hline
\end{tabular}

Table 2. Decrease of the size of $R M_{\alpha}$ (relative to the size of the ventricles)

\begin{tabular}{||c||c|c|c|c|c|c|c|c|c|c|c|c|c|c|c|c|c|c||}
\hline Electrode & 1 & 8 & 15 & 22 & 30 & 37 & 44 & 51 & 58 & 65 & 69 & 73 & 78 & 84 & 92 & 100 & 108 & 114 \\
\hline \hline$R M_{0.1}-R M_{0.2}(\%)$ & 30 & 9 & 12 & 20 & 31 & 18 & 20 & 20 & 18 & 16 & 15 & 10 & 2 & 1 & 5 & 9 & 11 & 47 \\
\hline$R M_{0.2}-R M_{0.5}(\%)$ & 15 & 65 & 60 & 51 & 31 & 34 & 22 & 21 & 21 & 21 & 21 & 26 & 10 & 6 & 12 & 12 & 27 & 24 \\
\hline
\end{tabular}

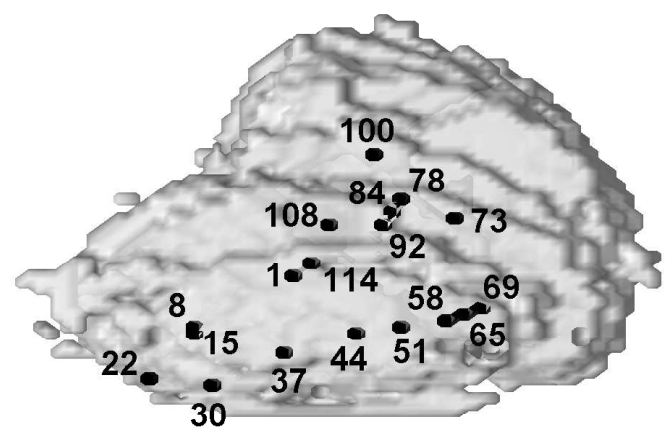

Fig. 4. Locations of the centers of $R M_{0.5}$ in the ventricles for every electrode

variation of the size of $R M_{\alpha}$ for increasing $\alpha$ is, in general, larger for electrodes positioned on the anterior chest, as shown in Table 2. If we compare once again electrode 84 to electrode $37, R M_{0.1}-R M_{0.2}$ is for the former $1 \%$ whereas for the latter is $18 \%$.

The locations of the centers of the $R M_{0.5}$ for every electrode are shown in Figure 4. Not surprisingly, they are distributed following the locations around the thorax of the corresponding electrodes. These results confirm, therefore, that 
electrodes on the anterior (posterior) chest focus on anterior (posterior) walls of the ventricles. Noticeably, the centers of electrodes on the anterior chest are closer to the periphery of the ventricles. Again, this observation can be explained by the relatively smaller size of $R M_{0.5}$ due to the distance separating skin positioning from the ventricles.

\section{Discussion}

The elucidation of the resolution properties of surface ECG systems can provide us with valuable clinical information, since it allows us to map patterns observed in ECG signals to the regions within the heart that generate them. In this study we combined bioelectric signal modeling and numerical methods to explore the notion of resolution, as expressed in the concept of RM. We defined the RM in terms of the $M S D_{n}$ resulting from approximating at the recording site, the signal induced by the whole source to the signal induced by the RM. It is, thus, a measurement that has a clear physical meaning and, since it is defined over recorded ECG signals, it is suitable to carry out fair comparisons of the resolution properties of different surface ECG systems.

Numerical methods proved to be the best choice to study the RM, as compared to experimental settings and other analytical techniques. Numerical approaches have, however, general limitations. Since an underlying model is assumed, the significance and generality of the results depend considerably on the ability of the model to describe the physical phenomena under investigation. In this study we have described the conducting properties of the body based on the VHM dataset, and the electrical activity of the heart based on a state machine model. Besides, the dynamics of the sources elements have been generated by simulating the stimulation of the apex at 60 beats per minute. In order to investigate the impact of the choice of the signal model on the scope of the results, in the future we will implement different thorax models and simulate cardiac electrical activity based on other descriptions and stimulation protocols.

In an application example, we showed that the RM in the ventricles depends on the positioning of surface electrodes. Specifically, we showed that both the location and the size of the RM depend on the proximity of the electrodes to the ventricles and on their angular coordinate around the thorax. Our results confirm that, by appropriately positioning the electrodes we can increase the amount of signal from a region of interest within the heart, although there are limits to the ability of an electrode to focus on selected regions smaller than the RM. By placing the notion of resolution in a quantitative framework, this approach complements other experimental and clinical settings intending to establish the limits of the electrocardiography [9]. The determination of the resolution properties of electrode systems can allow us to assess the convenience of standard electrode positioning and to explore new positioning for extracting the maximum amount of physiological information from the ECG. Thus, a quantitative definition of optimal electrode placement can be achieved based on the concept of RM. 
In summary, the combination of bioelectric signal modeling and numerical simulations constitute a powerful method to study the resolution properties of ECG systems. Firstly, they provide the formulation needed to estimate the RM. Key features of the RM include its location and its size, from which we can establish limits to the ability of the electrode system to focus on selected regions within the heart. Secondly, it forms a framework to assess, by implementing different anatomical models, the influence on the resolution properties, of factors often regarded as sources of variability, such as the shape of the body, the proportion of tissues or the size of the heart. Thirdly, complete multielectrode systems and varied electrode configurations can be systematically studied and compared, in order to determine optimal electrode placements. Numerical approaches to the resolution can complement other experimental and clinical settings, both as a means of explanation and of future experimental design.

Acknowledgments. This work has been partially supported by a Research Grant from Guidant Spain, and from the Research Project GR/SAL/0471/2004 from Comunidad de Madrid. Jesús Requena-Carrión is supported by grant 01836-04 from Consejería de Educación de la Comunidad de Madrid.

\section{References}

1. Lux, R., Bilbao, M., Pelter, M., Fleischmann, K., Zegre, J., Schindler, D., Drew, B.: Optimal Leads, Estimation, and Continuous Monitoring Improve Detection of Acute MI and Transient Ischemia. J. Electrocardiol. 37 Suppl, 240-243 (2004)

2. Trägård, E., Engblom, H., Pahlm, O.: How many ECG leads do we need? Cardiol. Clin. 24, 317-330 (2006)

3. Kornreich, F., Montague, T.J., Rautaharju, P.M., Block, P., Warren, J.W., Horáček, B.M.: Identification of Best Electrocardiographic Leads for Diagnosing Anterior and Inferior Myocardial Infarction by Statistical Analysis of Body Surface Potential Maps. Am. J. Cardiol. 58, 863-871 (1986)

4. Kornreich, F., Montague, T.J., Smets, P., Rautaharju, P.M., Kavadias, M.: Knowledge-Based System for Classification of Body Surface Potential Maps. In: Computers in Cardiology pp. 479-482 (1990)

5. Horáček, B.M., Warren, J.W., Penney, C.J., MacLeod, R.S., Title, L.M., Gardner, M.J., Feldman, C.L.: Optimal Electrocardiographic Leads for Detecting Acute Myocardial Ischemia. J. Electrocardiol. 34 Suppl, 97-111 (2001)

6. Malmivuo, J., Plonsey, R.: Bioelectromagnetism: Principles and applications of Bioelectric and Biomagnetic Fields. Oxford University Press, New York (1995)

7. Alonso Atienza, F., Requena Carrión, J., García Alberola, A., Rojo Álvarez, J.L., Sánchez Muñoz, J.J., Martínez Sánchez, J., Valdés Chávarri, M.: A probabilistic model of cardiac electrical activity based on a cellular automata system. Rev. Esp. Cardiol. 58(1), 41-47 (2005)

8. Kauppinen, P., Hyttinen, J., Heinonen, T., Malmivuo, J.: Detailed model of the thorax as a volume conductor based on the visible human man data. J. Med. Eng. Technol. 22(3), 126-133 (1998)

9. MacLeod, R., Kornreich, F., van Oosterom, A., Rautaharju, P., Selverster, R., Wagner, G., Zywietz, C.: Report of the first visualization of the reconstructed electrocardiographic display symposium. J. Electrocardiol. 38, 385-399 (2005) 